\title{
GUI based Industrial Monitoring and Control System
}

\author{
${ }^{1}$ Gaurav V. Barmase, ${ }^{1}$ Gaurav V. Khopade, ${ }^{1}$ Shital P. Thawkar, \\ ${ }^{1}$ Sahil P. Bawankule, ${ }^{1}$ Nikhil D. Gajbhiye, ${ }^{1}$ Shailesh Sakhare \\ ${ }^{1}$ Electronics and Telecommunication Engineering, \\ JD College of Engineering and Management, \\ Nagpur, India.
}

Abstract: - The main aim of this project is to develop a Graphical User Interface (GUI) based system to monitor and control the industrial process. The proposed protocol os user-friendly and it is more efficient due to the incorporation of a simple GUI. Moreover, the proposed system is installed to collect the valuable information.

Keywords: Graphical User Interfaces [GUI], industrial control, visual indicators, sensors.

\section{INTRODUCTION}

The concept of HCI (Human Computer interaction) came early 60's by developing a new idea of Graphical User Interface. Now researchers have reached a point where, in our daily lives, a Graphical Windows have been developed to make industrial work easier and faster with a single click of a button. Nowadays GUI are used in applications such as industrial manufacturing process, home automation etc. There are several Graphical software applications are available in the field of computer science or in electronics like python, Visual Basic, C\#, Linux etc., that integrate GUI (Graphical User Interface) and their programming scripting function for the interface designer and software developer to work easily on a software development work.

In this project, the complete Graphical User Interface [GUI] system is proposed, where it can be used to control and monitor industrial devices by using Python Tkinter and control system is interfaced with the computer through USB to establish a serial communication

ISSN: 2582-418X

Submitted: 26.04.2021

Revised: 19.05.2021

Accepted: 7.06.2021

Published: 14.06.2021
108

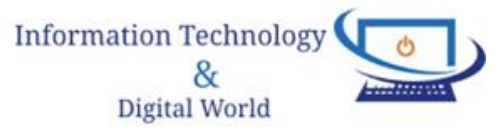


Journal of Information Technology and Digital World (2021)

Vol. 03/ No. 02

Pages: 108-117

https://www.irojournals.com/itdw/

DOI: https://doi.org/10.36548/jitdw.2021.2.004

\section{LITERATURE SURVEY}

Siemens-7SJ series: The Siemens 4 7SJ61 relays can be used for line protection for high and medium voltage networks with earthed (grounded), low resistance earthed, isolated or compensated neutral point. When protecting monitors, the SIEMENS 4 7SJ61 is suitable for asynchronous machines of all sizes. The relay performs all functions of backup protection supplementary to transformer differential protection [1].

Areva MICOM Series: MiCOM alstomP543-P546 is a range of transmission class protection relays pro-viding high speed current differential unit protection. They are designed for all overhead line and cable applications, interfacing readily with the longitudinal (end-end) communications channel between line terminals. The interface options support direct fiber optic, or multiplexed digital links [2].

Vamp: VAMP 245 feeder and motor manager is optimized to protection applications where directional earth fault and residual voltage stages are required in addition to standard over and residual current protection elements. The hardware supports eight digital inputs and seven outputs. Similarly, there are other manufacturers like Schneider, Texas, Toshiba etc.1973- The Alto, the first computer to have a modern graphical user interface, is born at Xerox PARC [3].

ABB-REF Series: REF610a is primarily intended forth protection of incoming and outgoing feeders in distribution substations of resistance earthed and solidly earthed power systems. REF610 is suitable for employment in marine and off-shore environments. Supplied with an optional arc protection function, REF610 also provides fast substation bus bar arc-fault protection. REF610s are also used for back-up protection of motors, transformers and generators to increase protection redundancy in critical utility and industrial applications [4].

International Journal of Software Engineering \& Applications (IJSEA), Vol.3, No.1, January 2012 140 Model Based Testing (MBT) is a quick and organized method which automates the testing process through automated test suite generation and execution techniques and tools [5]. Basili, Victor R., et al put forth a "TAME project" to enhance the software ecosystem. Korel, Bogdan [6].

Information Technology

\&

Digital World 
Journal of Information Technology and Digital World (2021)

Vol. 03/ No. 02

Pages: 108-117

https://www.irojournals.com/itdw/

DOI: https://doi.org/10.36548/jitdw.2021.2.004

Further in [7] authors designed a method to generate the test data using the software that is automated employing the "alternate variable method" that closely resembles the "hill climbing."

Gendreau, Michel, et al [8] designed an innovative "insertion as well as post optimization procedure" for the TSP that takes only the candidate solutions into consideration. Further, Basili, Victor R., et al [9] put forth a "TAME project" to enhance the software ecosystem. Korel, Bogdan. Et al [10] designed a method to generate the test data using the software that is automated employing the alternate variable method that closely resembles the hill climbing. Reau, Michel, et al [11] designed an innovative insertion as well as post optimization procedure for the TSP taking into consideration only the candidate solutions that are nearby. McMaster, et al [12] conducted the "Call-stack coverage for GUI test suite reduction.

Praditwong, et al [13] performed the Software module clustering as a multi-objective search problem. Whereas, Ding et al [14] put forth the automatic generation of software test data based on hybrid particle swarm genetic algorithm. Singh et al. [15] created an integrated strategy to accomplish suite selection using an ACO and a genetic algorithm. Arcuri, et al [16] devised a hitchhiker's guide to incorporate statistical tests for assessing the randomized algorithms in software engineering.

\section{PROPOSED METHOD}

Concept of GUI based control and monitoring system is still under progress, where some amount of work is completed but some projects are only accessible at the college level. The primary aim of this project is to implement the prototype of this project at college level. The concept of GUI based industrial monitoring and control system is the upcoming need for industrial World. It will help to improve the quality, save the time and make the industrial systems more effective. This system will automatically control and monitor industrial applications and generate alert to show the situation of appliances to take intelligent decision using this concept of GUI.

1. PYTHON was utilized as the software in the proposed work. IDLE stands for graphical user interface in python development. In other words, IDLE is a graphical user interface that allows us to edit, execute, browse, and debug python programs all from a single interface.

Information Technology $\&$ Digital World 
Journal of Information Technology and Digital World (2021)

Vol. 03/ No. 02

Pages: 108-117

https://www.irojournals.com/itdw/

DOI: https://doi.org/10.36548/jitdw.2021.2.004

2. In this project a complete industrial GUI system is proposed, where GUI is developed using python interface. TK is the combination of python and Tkinter, which provide easy as well as faster way to create GUI application.

3. The proposed work aims to monitor the temperature, pressure and vibrations coming from industrial machinery. Arduino IDLE is the software that helps to interface the sensors.

4. Data acquisition plays an important role in industries to ensure the quality of service. All this above will help to make the software more effective and efficient.

\section{A. Working}

As our project based on the Industrial monitoring and Control system. So, this project is divided into two parts, where the first is software based GUI and second is the hardware part. In the software based GUI, various types of Industrial process like Temperature, Utility, Pressure and Vibration of Machines, Oil level are shown. For checking this various types of process, various buttons are given on our Graphical User Interface [GUI] and all buttons have its own and different works according to the user needs. By clicking on the button, we can monitor and control the different processes. After selecting the above option, you will see a message on the screen while checking the process as shown in the below figure. You can click the stop button after the process is checked. If it is required to check another different process, it will click on the back button and check another process. The hardware part of this project aims to monitor and control the two major problems occur in the industries i.e. Temperature monitoring and control and the second is oil level of machines for better and non-stop production of industries.

The proposed project utilizes ESP32 module for wireless communication, HC SR04 ultrasonic sensor for checking the oil level, and NTC thermistor temperature sensor for checking the machine temperature. The ESP-2 includes two processors. The Wi-Fi and Bluetooth both are built in pin no. 30 or 36 according to user interface.

HC-SR 04 ultrasonic distance sensor and SONAR are used for detecting the distance to an object. This module includes an ultrasonic transmitter, receiver and control circuit i.e. Power (VCC), Trigger (Trig), Receiver (Echo), and ground (GND).

ISSN: 2582-418X

Submitted: 26.04.2021

Revised: 19.05.2021

Accepted: 7.06.2021

Published: 14.06.2021

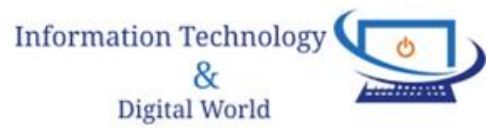


Journal of Information Technology and Digital World (2021)

Vol. 03/ No. 02

Pages: 108-117

https://www.irojournals.com/itdw/

DOI: https://doi.org/10.36548/jitdw.2021.2.004

The NTC Thermistor temperature sensor module is three pin modules. NTC is very sensitive to ambient temperature. The temperature detection range of the module is between 20 degrees Celsius to 80 degrees Celsius. It works on $3.3 \mathrm{~V}$ to $5 \mathrm{~V}$ voltage.

The proposed project has connected the HC-SR04 ultrasonic sensor to the ESP 32 module by connecting the VCC pin to $3.3 \mathrm{~V}$ on the ESP32 and GND pin to ground on the ESP32 and Trig pin to D2 on the ESP32. Similarly, connecting the Echo pin to D5 on the ESP32 is possible.

In this, the ultrasonic sensor is mounted to the top of the oil container for measuring the oil level of the container. When the oil level drops below a specific level, the sensor detects it and sends an alarm to the control system, instructing it to refill the tank. The same data will be shown on our GUI-based monitoring system. Similarly, the NTC thermistor temperature sensor is also connected to the ESP 32 by connecting the A0, D0, VCC and GND pin of the sensor to ESP32 module. This NTC sensor is connected to the machine for checking the machine temperature and through ESP module it will send the data to the control system and also shows temperature on our GUI based monitoring system. If machine temperature increased above the specific range, then it will send the alert and control system to stop the working of the machine. By using this project, machine production will be increased and it saves time. Cost will also reduce the human efforts and it improves the productivity and quality of work.

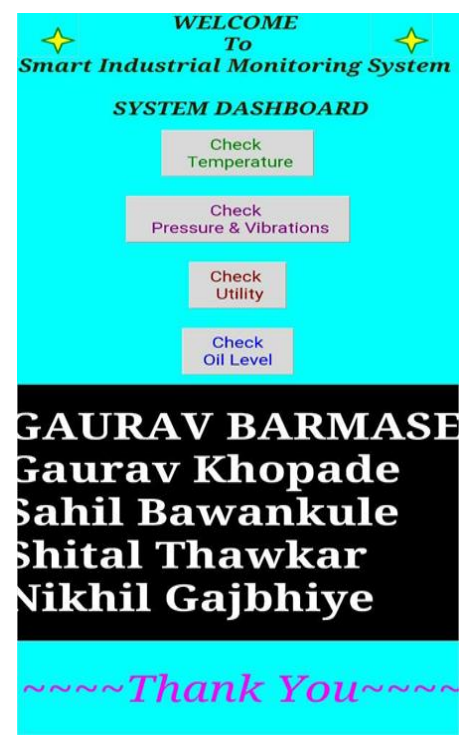

Fig1: Screenshot of Working Module

ISSN: 2582-418X

112

Submitted: 26.04 .2021

Revised: 19.05.2021

Accepted: 7.06.2021

Published: 14.06.2021

Information Technology

\&

Digital World 
Journal of Information Technology and Digital World (2021)

Vol. 03/ No. 02

Pages: 108-117

https://www.irojournals.com/itdw/

DOI: https://doi.org/10.36548/jitdw.2021.2.004

The process of smart industrial monitoring system starts with the main page of the portal; it consists of various buttons as shown in fig. 1. Every buttons have individual work as per the design of the page.

When we click on start button will get first page shown in fig.1. Then next button is the checking various different processes i.e. Temperature, utility, pressure and vibration, etc. The button can monitor and control different processes.

After selecting the above option, a message will be displayed on the screen while checking the process as shown in the fig.1. The stop button can be clicked after the process is checked. If it is required to check another different process, the back button is clicked and it will check another process.

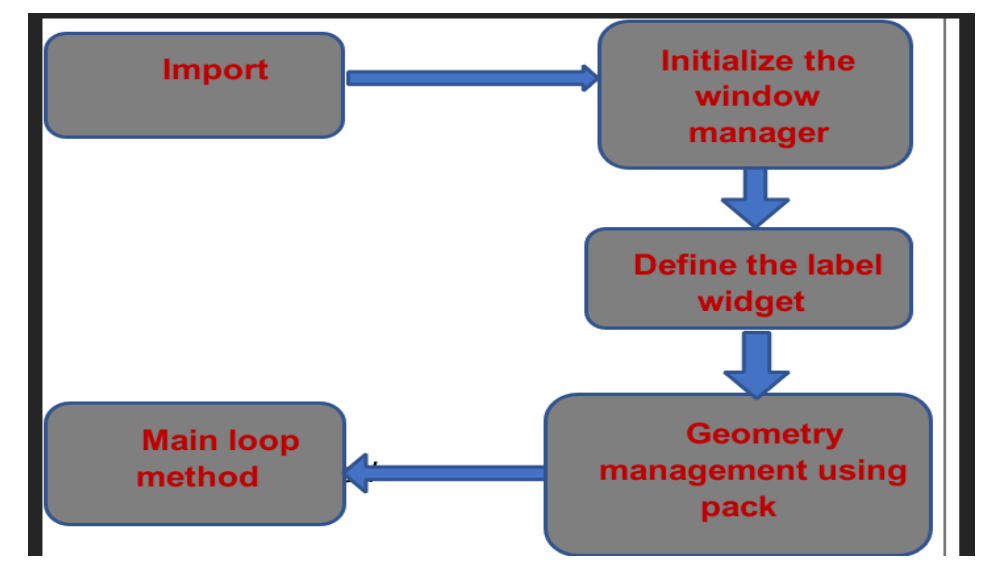

Fig2: Flow Chart of GUI System

Tkinter - It is based on Python interface to the Tkinter Graphical user interface toolkit. Tkinter is the standard GUI library for Python. Python when combined with Tkinter provides an easy way to create Graphical User Interface [GUI] application. Tkinter provides a powerful object-oriented interface to the Tkinter Graphical User Interface [GUI] toolkit.

We need to perform the below steps:

1. Import the Tkinter modules.

2. Create the Graphical user interface application window. 
Journal of Information Technology and Digital World (2021)

Vol. 03/ No. 02

Pages: 108-117

https://www.irojournals.com/itdw/

DOI: https://doi.org/10.36548/jitdw.2021.2.004

3. Add one or more of the above given widgets to the Graphical User Interface [GUI] application.

Tkinter provides multiple controls such as labels, buttons, and text boxes used in a Graphical User Interface [GUI] application. These controls are commonly called as widgets.

4. Geometry Management All Tkinter widgets have access to specific geometry management methods, which have the purpose of organizing widgets throughout the parent widget area.

5. Then it enters the main loop to take action on each event triggered by the users.

\section{B. Technologies used}

1. Python

2. Tkinter

3. ESP32

\section{Results}

Most important part of the system is result since it measures the selected process and shows the reading and then the process can be controlled.
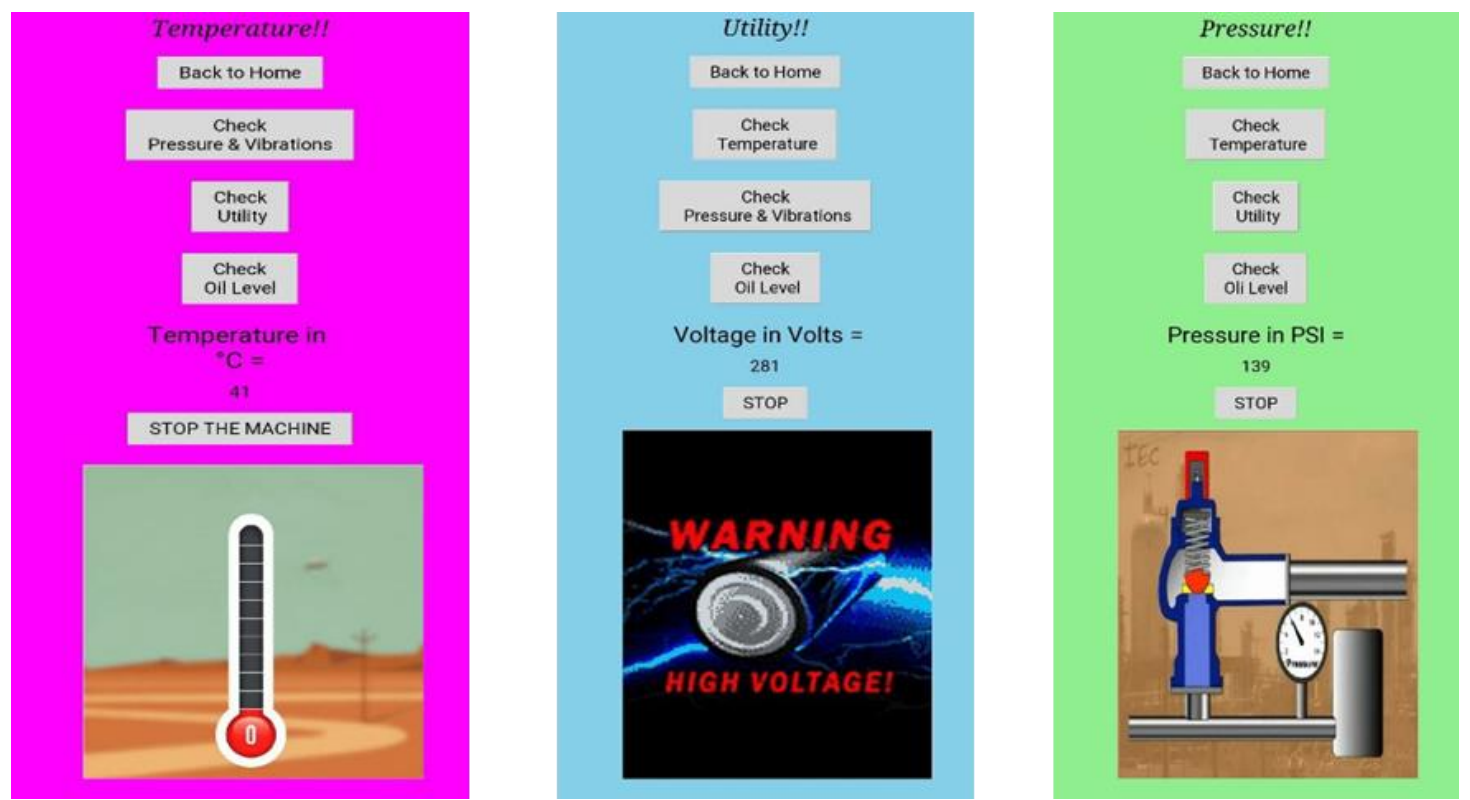

Fig3: Screenshots of Proposed GUI System 
Journal of Information Technology and Digital World (2021)

Vol. 03/ No. 02

Pages: 108-117

https://www.irojournals.com/itdw/

DOI: https://doi.org/10.36548/jitdw.2021.2.004

\section{CONCLUSION:-}

The system is designed to increase the safety and lifetime of the machine. The efforts of the research study can successfully monitor and operate industrial equipment by employing the proposed system and software.

\section{FUTURE SCOPE:-}

This system can be updated as per their industrial requirements. Some more different processes can also be integrated as per the requirements of the industry.

\section{ACKNOWLEDGMENT:-}

We all the team members specially want to thank our project guide Prof. Shailesh Sakhare and Prof. N. N. Gyanchandani for his informative and motivational guidance.

\section{REFERENCES}

[1] Marc Lavergne, "Graphical User Interface for Next Generation Power Systems", Twentysecond International Telecommunications Energy Conference, 2000. INTELEC, pp. $109-112$.

[2] FGlori, O. N. Hemming, A. Luchetta, G. Manduchi, V. Schmidt2, C. Talierci03, S. Vitturi, "Graphical User Interface of RFX Control and Data Acquisition System", 15th IEEEINPSS Symposium on Fusion Engineering, 1993., (Volume:2), pp. 577-580

[3] Jorgensen, A. H. and Myers, B. A. "User interface history". Proceeding CHi '08 extended abstracts on Human factors in computing systems, 2008, pp. 2415-2418.

[4] Harnani Hassan, Raudah Abu Bakar, Ahmad Thaqib Fawwaz Mokhtar, "Face Recognition Based on Auto-Switching Magnetic Door Lock System Using Microcontroller ", 2012 International

[5] Conference on System Engineering and Technology September 11-12, 2012.Gurinder Singh, Lakshmi Munukutla, " Interfacing USB Printer using Vinculum Host Controller", American Society for Engineering Education, 2009.

Information Technology

$\stackrel{\&}{\text { Digital World }}$ 
Journal of Information Technology and Digital World (2021)

Vol. 03/ No. 02

Pages: 108-117

https://www.irojournals.com/itdw/

DOI: https://doi.org/10.36548/jitdw.2021.2.004

[6] Soumyajit Datta, Sumana Chowdhuri Jitendranath Bera "Development of USB compatible PC based PD motorcontroller for chemical process", 2012 1st International Conference on Power and Energy in NERIST (ICPEN), pp. 1 - 4.

[7] Gonzalez, M.F.O, Arizaga, S.1A. ; Moreno, B.O., "Temperature-Meter via USB Base Don PIC 18F2550 for Solar Energy Concentrator System", International Conference on Electrical, Communications, and Computers, 2009, CONIELECOMP 2009.pp. 1 - 7.

[8] Junqin Wang," Design Intelligent Temperature Monitoring System Based on DSP", 2012 4th International Conference on Intelligent Human-Machine Systems and Cybernetics, pp. 234237.

[9] Zhou Runjing, Xu Hongwei, Ren Guanzhong, "Design of Temperature Measurement System Consisted of FPGA and DSI8B20", 2011 International Symposium on Computer Science and Society, pp. 90-93.

[10] Andisheh Feizil, Chui Yin Wong, " Usability of User Interface Styles for Learning a Graphical Software Application ", 2012 International Conference on Computer \& Information Science (ICCIS), pp. 1089-1094.

[11] Frank McKiel Jf. "Audio-Enabled Graphical User Interface For The Blind Or Visually Impaired", Proceedings of the Johns Hopkins National Search for Computing Applications to Assist Persons with Disabilities, 1992, pp. 185 - 187.

[12] Scott Koziol, Craig Schlottmann, Arindam Basu, Stephen Brink, Csaba Petre, Brian Degnan, Shubha Ramakrishnan, Paul Hasler, and Aurele Balavoine, " Live Demonstration: Hardware and Software Infrastructure for a family of Floating-Gate Based FPAAs " Proceedings of 2010 IEEE International Symposium on Circuits and Systems (ISCAS), pp. 2793.

Information Technology $\&$
Digital World 
Journal of Information Technology and Digital World (2021)

Vol. 03/ No. 02

Pages: 108-117

https://www.irojournals.com/itdw/

DOI: https://doi.org/10.36548/jitdw.2021.2.004

[13] Yu-Chen Chen, Yuan-Hsiang Chang, Te-Chuan Wang, "A Real-Time Computer Graphical User Interface for Advanced Boiling Water Reactor Case Comparison Using MAAP Software", Computer Symposium (ICS), 2010 International IEEE conference, pp. 274 - 278.

[14] Marc Lavergne, "Graphical User Interface for Next Generation Power Systems", Twentysecond International Telecommunications Energy Conference, 2000. INTELEC, pp. 109 - 112.

[15] Vijayakumar, T., Mr R. Vinothkanna, and M. Duraipandian. "Fuzzy Logic Based Aeration Control System for Contaminated Water." Journal of Electronics 2, no. 01 (2020): 10-17.

[16] Jain, Sukrutha A., and Avinash Bharadwaj. "Characterizing WDT subsystem of a Wi-Fi controller in an Automobile based on MIPS32 CPU platform across PVT." Journal of Ubiquitous Computing and Communication Technologies (UCCT) 2, no. 04 (2020): 187-196.

[17] Shakya, Subarna. "Analysis of Soil Nutrients based on Potential Productivity Tests with Balanced Minerals for Maize-Chickpea Crop." Journal of Electronics 3, no. 01 (2021): 23-35.

[18] Sungheetha, Akey, and Rajesh Sharma. "Fuzzy Chaos Whale Optimization and BAT Integrated Algorithm for Parameter Estimation in Sewage Treatment." Journal of Soft Computing Paradigm (JSCP) 3, no. 01 (2021): 10-18.

[19] Chen, Joy Iong-Zong, and Lu-Tsou Yeh. "Greenhouse Protection Against Frost Conditions in Smart Farming using IoT Enabled Artificial Neural Networks." Journal of Electronics 2, no. 04 (2020): 228-232.

Information Technology $\stackrel{\&}{\text { Digital World }}$ 